\title{
Semantic Interpretation of English Nouns Referring to Units of Time
}

\author{
Natalia Gorskaya ${ }^{1, *}$, and Vera Glyzina ${ }^{2}$ \\ ${ }^{1}$ Irkutsk National Research Technical University, Institute of Linguistics and Intercultural Communication, 664074, Irkutsk, \\ Russia \\ ${ }^{2}$ Baikal State University, Foreign Languages Department, 664003, Irkutsk, Russia
}

\begin{abstract}
The paper deals with the semantics of English nouns integrating a time component basing on respected English-language dictionaries. The timeliness of the topic under consideration is substantiated in terms of interaction and interconnection of the language and culture and is proved to be one of the major problems of the cognitive linguistics. The nouns possessing the semantic feature of "time" in their meaning have been revealed and analyzed. The culture, customs and traditions of different nations are pointed out to influence directly the formation of the language worldview which is expressed in the meanings being different from each other. Nominal groups of time semantics are shown to be characterized by sufficient amount of possibilities to express time meanings. A group of nouns in the English language has been proved to represent a single semantic structure of the lexical item of time. This lexical item is classifiable and can be identified from personal acquisition. The results of the investigation are theoretically significant covering the general doctrine of words and their semantic structure. The results, in particular, indicate that a considerably greater number of semantic features is available in the peripheral sphere of a noun meaning than it was used to be believed, the same being true of the prototype nature of these very features. All these factors are of apparent interest concerning any investigation in the field of cognitive science. Practical importance of the work consists in the possibility to use the results of the investigation in the English language practical training, in the lecture courses dealing with lexical semantics, cognitive linguistics, while compiling educational books, instructing on term papers and undergraduate theses.
\end{abstract}

\section{Introduction}

The problem of time has been investigated by the scientists belonging to different fields over a great number of years. In modern linguistics, the correlation of cognitive processes and the meanings of linguistic units is responsible for this interest and, in particular, for the subject matters involvement into the temporal exposition structure. It has been pointed out by investigators that temporal meanings can be expressed not only by means of verbal forms, but in many other ways too, for example, by adverbs, objective complements of time, date indications, etc. [1], [4]. The investigation of temporal dictionary from the point of cognitive linguistics view is a model expressing definite ideas of a human with regard to some phenomenon. The idea of "time" is considered to be a certain real time reflection in a language, the real time being closely connected with extralinguistic activities of a person through the acquisition of, first of all, the spatial nature (objects, places). As it is indicated that time imagery reflects the rhythms and paces of the society development in addition to its numerous living orientations. Time is one of the most important constituents of human self-presence, the constituent mentioned being an interesting objects to study as it is based on many sciences in view of manifestation omnitude and exceptional nature of its properties. In many scientist' opinion, the daily experience is enough for absorbing the theory of the time nature. Nevertheless, it is evident that neither real physical time (which is a subject matter in natural sciences), nor psychological phenomenon of" the time being lived" that is "time of life being lived through" does not appear to be a genial object of a linguistic survey, although they are directly relevant to the language. The "time of life" can be referred to the sphere of self-awareness of a human, this being one of the main forms for a human to "attain the world around". Probably, the link of time and an intellectual coordinate has its etymological backing too. According to other scientists there are etymological parallel guides between the lexical-semantic complexes of "time" and "sense". They referre time to the categories that are not only described by means of a language, but are also found in it, in its dictionary and grammar structure. As for the linguistic aspect of time, usually the notion of "time" is considered to be a reflection of some real time outline in the language. The language is known to form a continuum with other forms of a human behavior.

* Corresponding author: natgo2012@yandex.ru 
Therefore, the category of time in the language can be referred to such notions which can be used to determine the correlation of linguistic activities with an extralinguistic one of a human being and which contribute to transforming the real world into a "projected world" of a human consciousness. So, if event specifications of quantity are found as basic ones in building physical time outlines, in building linguistic time outlines specifications of quality are in predominance to deal with temporal relations. It should be noted that the problem of a language representation of time in the linguistics is most frequently connected with the temporality and aspect of a verb, as the verb essentially means an activity in the broad sense of the word, an activity always running in the time. Thus, as it is possible to reach the agreement with $\mathrm{B}$. Rassel, who making reference to the definition of physical time (object and object) sets it in opposition to mental time (subject and object) [12].

\section{Semantic theory of V.G.Gak}

Many native language experts, some of them being quite famous, such as N.D. Arutyunova [1], Yu.N. Kara'ulov[8], V.G. Gak [5], A.Ya. Gurevich [6], M. Makovskiy [14], T.V. Bulygina [3], etc. dealt with the subject of time semantics nature. Our investigation of time perspective semantics with regard to nouns is based on the theory formulated by V.G. Gak on semantic matching as a formal facility to organize an utterance. In accordance with the above theory, the matching noun semes enable the semantic matching theory and the evidence of a temporal seme availability contained by a noun to be used in the material being investigated. V.G.Gak gives the following definition of seme: "The semantic structure of word meaning is the totality of elementary senses - semes". V.G.Gak worked out a hierarchial system of semes in the word meaning. He singled out the archiseme which expresses the generic meaning: e.g. wolf - a living being; to come - an action; table - an object; beautiful - a quality. The second group of semes is made up of differential semes which carry some additional information, modify or qualify the idea expressed by the archiseme: e.g. spinster may be split into the following semes: 1) a human being (archiseme); 2) female; unmarried; elderly (differential semes). There may be several differential semes in the word meaning. V.G.Gak distinguishes differential semes of two types: 1) descriptive, reflecting the qualities of the object which are revealed through the opposition of lexical units in terms of differences and similarities (male / female; married / unmarried); 2) relative, reflecting relations between different objects - functional, temporal etc. The third group is represented by potential semes which are actualized in the word meaning under the influence of a certain context. e.g. cat does not contain in its semantics such semes as "scratchy", "purring", "gentle", "soft" etc. These semes are made prominent in some particular contexts; on these grounds they are referred to potential semes. We also support the J. Miller and F. JohnsonLaird's point of views with regard to the concept that awareness of "time" is characterized by such major categories as short time intervals, prolonged and simultaneous ones and events that pertain to the past, present and future [15]. We can also talk about the primary or the nearest word meaning has a common use popular with people and enables the mutual understanding in human intercourse. Primary word meaning calls the above-mentioned meanings formal and content-related. The nearest word meaning calls all the subsequent meanings and indicating that all the other meanings are person-related in understanding though different in the quantity and the number of elements. Also the nouns used to express periods of time, that they can also define something which is not available in nature but which is used by the humanity in its life and activities. People live in real given conditions, that' why worldview in their consciousness is expressed by their language in the form of various meanings differing from each other. This fact is connected not only with the influence of culture, habits and traditions of the nation on the formation of a language worldview, but also with the specific life conditions of a particular language molders. The study of lexical formatives of every national language contributes to getting precious knowledge of the nation and its language worldview. Taking into consideration everything above mentioned, we can tel that person-related understanding viewed as an advanced scientific idea turns into a scientific definition resulting from a precise arrangement of use based on people's reasoning and in correspondence with the rules of logic. Different features are registered and fixed in one and the same object due to people's experience. This phenomenon takes place in correspondence with the viewpoint of the modern cognitive linguistics.

\section{Time period indicators}

The semantics of English nouns possessing a time component is given consideration to in the paper presented. The respected English language dictionaries of Hornby, LDCE, OED, WNCD, etc. were the main sources of the material collected. To define the name of time, more than 17 meanings are in store in the dictionaries mentioned including the ones that characterize its different kinds of styling. The empiric study shows that nominal groups possessing time semantics have enough possibilities to express their time meanings. Different time units can express a point in the time or a precise (sometimes not very precise) period of time and belong to the above groups possessing time semantics. For example: moment, instant, second, minute, hour, day, night, week etc. Thus, in the examples given, A.V. Kravchenko defines a second as a part of a 24-hour period, whereas a 24-hour period is an average period of time which is enough for the Earth to make one revolution around its axis. However, the fact of the Earth revolving around the Sun being unknown to the humanity, the latter was well acquainted with its effects and different language facilities to express it long prior the modern scientific worldview was created [12]. This point of view is shared by I.A. Klimishin. In his opinion, it is a cyclical nature and repeated regular motions of the Sun performed in the 
heavenly dome that have been used to measure the humanity being duration [10]. A.G. Gurochkina has come to the conclusion that the nominal groups of temporal semantics are divided by objective time into direct naming units of different amounts in spite of the fact that temporal activities are the primary function of these nominal groups [7].

Let us give our consideration to some examples. Some time period indicators such as minute and second are coordinated by native speakers with an amount of time. However, as it is indicated by S. Yakovleva, our intellect allows these lexical units to have the second meanings which are not connected with the scientific categories of time while being connected with the idea of a life time. This time interval can be identified like any category of our existence and is possible to be assessed and described [18]. In this occasion, the nouns of minute and second are close in their meaning to such words as moment, instant which, however, do not belong to common units of time measurement but are only conventional signs to be used in the cases when an event is not fixed. These words can be interchangeable but never synonymous. The noun of moment is used to describe time regardless of any specific physical units of measurement. The above-mentioned noun can be used in speaking to denote the noun of hour as the shortest period of time. In all the dictionaries, the primary meaning of the noun of hour denotes a time period of 60 minutes duration. Its secondary meaning is semantically somewhat different in various dictionaries. However, the opinions of lexicographers vary when the third, fourth, fifth, sixth and the successive meanings are compared. This is particularly true with regard to the arrangement order of the noun of hour definition denoting a time period and to the defining features of the noun hour meaning. Thus, for example, in the LDCE hour is given the definition of a distance covered in this period of time, this being the third meaning, whereas in the WNCD this meaning is the sixth in the order. The fourth meaning of the noun of hour in the LDCE and OED is a definite time period or a fixed time, whereas in the Hornby dictionary this meaning is the third in the order. In some dictionaries there are also attributive modifiers typical for astronomical and geographical meanings of the noun of hour. In the OED, this meaning is arranged the seventh, while in the WNCD it is the eighth in sequence. It should be noted that lexicographers are about to share the opinion concerning the defining features of astronomical and geographical meanings of the noun of hour, their additional ideas being different and insignificant in each case. As for LDCE and Hornby, this meaning is not identified in these dictionaries. As for the noun of day and night, the agreement of attributive modifiers is revealed with regard to the first meaning in all the dictionaries. The following definitions are given by lexicographers: time, time reference, time period. Further, their opinions differ and these nouns have different meanings in all the dictionaries. For example, the noun of day in the meaning of the period of work takes the fifth place in the LDCE, it is the sixth in the order in the OED, while in the WNCD it occupies the seventh place. The dictionary meaning of the noun of night is one and the same in all the dictionaries with regard to the first meaning, namely, the hours of darkness: they are the dark period of time from the sunset to the sunrise, that is the period of darkness. Hereafter, the opinions concerning the meanings vary in all the dictionaries. Basing on the abovementioned information, a conclusion can be made on the duplex structure of the time noun definition, its primary and subsequent meanings. In accordance with A.A. Potebnya's indications, the primary or the nearest word meaning has a common use popular with people and enables the mutual understanding in human intercourse [16]. S.D. Katsnelson calls the above-mentioned meanings formal and content-related, the second term covering all the subsequent meanings and indicating that all the other meanings are person-related in understanding though different in the quantity and the number of elements [9]. It should be noted that there is a point of view with regard to the nouns used to express periods of time, that they can also define something which is not available in nature but which is used by the humanity in its life and activities [13]. Considering that people live in real given conditions, the worldview in their consciousness is expressed by their language in the form of various meanings differing from each other. This fact is connected not only with the influence of culture, habits and traditions of the nation on the formation of a language worldview, but also with the specific life conditions of a particular language molders. It has been indicated by Ye.A. Shendeleva that the study of lexical formatives of every national language contributes to getting precious knowledge of the nation and its language worldview [17]. Thus, a person-related understanding viewed as an advanced scientific idea turns into a scientific definition resulting from a precise arrangement of use based on people's reasoning and in correspondence with the rules of logic [17]. N.S. Kovalenko emphasizes that different features are registered and fixed in one and the same object due to people's experience. This phenomenon takes place in correspondence with the viewpoint of the modern cognitive linguistics [11]. Thus, a group of nouns denoting a human in different periods of its biological development was identified. For example: in his childhood; the tension between his background and boyhood ... (FT, Sep. 7, 1998); time misspent in youth is sometimes all the freedom one ever has (CD, 1993); she must have watched Roots as a child (FT, Aug. 13, 1998). Onwards, the availability of temporal semantics was noted in identifying a noun at large in sentences with the noun of self. The constructions containing self and the adnoun of former enable to speak of a background period in a human life to compare it with the present moment. For example: I looked at the once lively rattle-pated, humorous little doctor ... and I saw nothing left of his former self, but the old tendency to vulgar smartness in his dress (Collins, 360); Shadow of his former self (RTE, 1987). Proper names can also vary as needed while a human advances his course of life. For example, Andy Andrew; Archie - Archibald; Bab - Barbara; Bel - Bella - Isabel - Isabella - Annabell - Arabella; Margie Margery - Margaret. It should be noted that functional meanings of nouns are divergent inwardly, which is conditioned both by their position inside the lexical system and the specificity of the object classes being 
denoted as a direct correlation with the practical life of the society is registered both in the law of its development and usage. The investigation also showed that it is easy to recognize the nouns that characterize a person by profession or position that record on permanent business of the person. Their links with the temporal characteristic are different, although the both of them are temporary properties being the features of a person. The first ones anticipate more permanent properties of an individual, whereas the second ones are less permanent; therefore in dictionary definitions, time as an identifier can go with an ambassador, an apprentice, an artist, a president, but not with a writer, a musician, a sculptor, etc. A close link with time can be followed with regard to the names of political leaders and statesmen, and the nouns denoting membership in a party and any other non-government organization. Thus, in dictionary definitions of such words as a president, a minister, a chancellor and the like one can find an indication of time, that is, when and what term individuals are elected or appointed for. In the text, next to the indication of a position, one can also find a time qualifier: Mr. Fisher, a Saurer's chief executive since late 1996; Sam Dash, then a council for the Senate Watergate committee; Salmon Shah, a member of last year's interim government (FT, Aug.13, 1998); Victor Chernomyrdin, Russia's acting Prime Minister (FT, Sep.7, 1998). A time link is revealed with regard to nouns denoting position and social rank. It should be noted that the nouns characterizing a person by means of the vocational aptitude (artist, actor, singer) link with a certain time with a greater difficulty. For example, a sentence of the type "Mr. X, then a singer" can be interpreted as" Mr. X worked on a permanent basis at la Scala: Mr. X, then a singer at la Scala. It is an interesting fact that in a speaker's consciousness the nouns denoting periods of a human life by the age stand side by side with the nouns denoting a person by the social rank, as they are also connected with definite periods. That is they are events in the life of a human (a teacher is opposed not only to his students but also to children, whereas the latter are opposed not only to grown up and elderly people, but also to book-keepers, lawyers and clerks).

\section{Conclusion}

Taking into consideration everything above mentioned, one can make the following conclusions:

1. Different semantic types of nouns show their potential time seme in different ways. Functional and relational nouns show the temporal semantics most distinctly, their bearers "changing" their roles with regard to social, political, age characteristics. Persons with a greater or smaller time "link" are opposed to each other inside these groups (writer vs. manager). Besides, the nouns are revealed, their referents being linked with some definite time moments when they are engaged in their activities. 2. The nouns determining the periods of human lives by the age stand side by side with the nouns identifying a person by its social function, public position. In this situation, the characteristic being "a period of human life journey", i.e. "a period of time" is a general basis for comparison.

A survey carried out in the investigation confirmed the availability of features in greater numbers in the sphere of nominating than it is believed. The survey also proved the prototype nature to be a semantic time characteristic.

\section{References}

1. N.D. Arutyunova, Language and Time $170-200$ (1997)

2. G. Budykina, Siberian Phylological Journal 3, 22-23 (2015)

3. T.V. Bulygina, A.D. Shmelyov, Pragmatics. Semantics. Dictionary making. Aspect, tense, person, mood 574 (1997)

4. O. Ducrot, T. Todorov, Encyclopedic dictionary of Language science (Oxford, Blackwell, 1987)

5. V.G Gak, The space of time. Logical analysis of a language. Language and Time (Indrik, Moscow, 1997)

6. A.Ya. Gurevich, Kategoriya srednevekovoi kultury. The category of Middle Age structure (Iscusstvo, Moscow, 1972)

7. A.G Gurochkina, Space and time, Materials of scientific conference (MSU, Moscow, 1997)

8. Yu.N. Rara'ulov, General and Russian ideography (Nauka, Moscow, 1976)

9. S.D. Katsnelson, The content level of a word, meaning and its naming unit (Nauka, Moscow, Leningrad, 1965)

10. I.A. Klimishin, Calender and chronology, (Nauka. Moscow, (1990)

11. N.S. Kovalenko, Vestnik of Tomsk State University. Phylology, language and culture 1, 35-41 (2013)

12. A.V Kravchenk, Interacademic collection of science papers (ISLU, Irkutsk, 1997)

13. G. Lakoff, M. Johnson, Metaphors we live by, (The University of Chicago Press, Chicago and London, (1980)

14. M.M. Makovskiy, J. Puhvel, Etymology dictionary. Voprosy yazykoznaniya 4, 128-139 (1993)

15. G. A. Mecke, I. Lee, J.R. Baker jr., M.M. Banaszak Holl, B.G. Orr, Eur. Phys. J. E 14, 7 (2004)

16. A.A. Potebnya, Imageless thought and a language (SINTO, Kiyev, 1993)

17. E.A Shendelyeva, Topical problems of Russian philology (ITU, Tomsk, 2000)

18. E.S Yakovleva, Fragments of the Russian linguistic world image (models of space, time, acquisition) (Gnosis, Moscow, 1994) 